\title{
Taizhou's COVID-19 prevention and control experience with telemedicine features
}

\author{
Cenyi Shao ${ }^{1, *}$, Shijian $\mathrm{Li}^{2, *}$, Feng $\mathrm{Zhu}^{3}$, Dahai Zhao ${ }^{4}$, Hui Shao ${ }^{3}$, Haixiao Chen $(\bowtie)^{3}$, Zhiruo Zhang $(\bowtie){ }^{1}$ \\ ${ }^{1}$ School of Public Health, Shanghai Jiao Tong University, Shanghai 200025, China; ${ }^{2}$ Department of Public Health, SUNY College at Old \\ Westbury, Old Westbury, NY 11568, USA; ${ }^{3}$ Taizhou Enze Medical Center (Group), Taizhou Hospital of Wenzhou Medical University, Taizhou \\ 317000, China; ${ }^{4}$ School of International and Public Affairs, Shanghai Jiao Tong University, Shanghai 200025, China
}

(C) Higher Education Press 2020

\begin{abstract}
The outbreak of coronavirus disease 2019 (COVID-19) has spread rapidly around the world. As of May 30, 2020, a total of 84568 confirmed COVID-19 cases have been recorded in China, with a mortality rate of approximately 5.5\%. Taizhou is a prefecture-level city in Zhejiang Province. A total of 146 cases were diagnosed in this epidemic, with a fatality rate of $0 \%$. This condition is due to the establishment of an "Internet +" diagnosis and treatment model based on online medical application (APP), telemedicine, WeChat service, and consultation hotline in Taizhou. Taizhou led in opening the "COVID-19 Prevention and Treatment Special Line" in China, which is conducive to pre-hospital screening, suppressing social panic, and clinical support. Hospitals also carried out related online lectures and popularization of science. We summarize Taizhou's COVID-19 prevention and control experience with telemedicine features, with a view to providing reference for the control of the epidemic at home and abroad.
\end{abstract}

Keywords COVID-19; telemedicine; prevention experience

\section{Introduction}

Taizhou, as a prefecture-level city in Zhejiang Province, is also one of key areas for the prevention and control of COVID-19 epidemic. A total of 146 patients were diagnosed with COVID-19 in Taizhou, and all have been cured and discharged from the hospital as of March 11, 2020. In the early stage, due to the complexity of the epidemic, it was difficult for the public to identify themselves, and the general public was generally nervous and anxious, lacking corresponding self-assessment guidance and psychological intervention. At the same time, designated hospitals are also under greater pressure for admissions. But the city has implemented various prevention and control measures to respond to COVID-19, e.g., strict urban control, taking the lead in intensive treatment of COVID-19 cases in the whole province, and comprehensively and rapidly deploying an Internet prevention and

Received May 25, 2020; accepted June 11, 2020

Correspondence: Haixiao Chen, chenhx@enzemed.com; Zhiruo Zhang, zhangzhiruo@sjtu.edu.cn

${ }^{*}$ Cenyi Shao and Shijian Li contributed equally to this manuscript. control system. In particular, Taizhou innovatively launched the "Internet +" online diagnostic and treatment network service and opened the "COVID-19 Prevention and Treatment Special Line" (COVID-19 Special Line) to give the public the corresponding professional consultation, follow-up, intervention, and guidance online. For confirmed patients, Taizhou integrates the city's highquality medical resources, centralizes all confirmed cases to Taizhou Public Health Medical Center, and implements refined treatment using the one person-one team and one person-one plan.

\section{Taizhou's Internet + COVID-19 prevention and control model}

Since the outbreak of the COVID-19, Taizhou Enze Medical Center (Group) has established an "Internet + COVID-19 Prevention and Control Practice Group" based on the timeliness, interactivity, and controllability of telemedicine. In this group, the medical department of the hospital develops a plan, which is then implemented by the Internet medical center. The information center maintains the platform, the call center mans the hotline, 
and the nursing department handles the follow-up feedback, in which cooperative personnel from the volunteer alliance participate together.

Telemedicine, as a supplementary means of medical services, has successfully played its role in online consultation and diagnosis and treatment when offline medical services entered the emergency state. Taizhou Enze Medical Center (Group) considered Taizhou Hospital and Enze Hospital as its core and built an "Internet +" diagnosis and treatment model based on online medical APP, telemedicine, WeChat service, and consultation hotline, which radiates to all 136 health clinics in towns and townships. Taizhou led in opening the COVID-19 Special Line in China, which aims for the complete prehospital screening and early detection and treatment.

Doctors from the Department of Respiratory Medicine, Infectious Diseases, Emergency Medicine, Critical Medicine, and other departments constitute Internet medical call center experts who are responsible for the hotline and provide $7 \times 24$ free services to the public. Patients consult online through text, images, video screens, and more. The hospital also organizes live online lectures by experts to promote popular scientific knowledge and healthcare skills to the public.

The nursing team tracks feedback from people using different consultation sources to ensure the diagnosis of epidemic, early detection of suspicious patients, and early treatment and recovery. The COVID-19 Special Line also added online services for resumption of work, combined with offline services, to solve practical enterprise problems and improved the awareness and ability of enterprise employees in the prevention and control of epidemics.

In terms of information technology, the Internet medical center integrates the electronic design of epidemiological survey, runs through the whole process of outpatient service and hospitalization, and develops multi-dimensional technology applications, such as public service, clinical application database, and management decision. The information center has added three new functions on the WeChat public account, namely, access to the surrounding epidemic prevention map, intelligent selfinspection, and real-time update of the epidemic situation, to allow the public to easily determine their health status and proceed to the nearest designated medical institution through Internet self-examination for diagnosis and treatment.

\section{Preliminary effectiveness of Taizhou model}

The Taizhou COVID-19 Special Line has been operating since January 23, 2020. As of March 15, 2020, at 24:00, the online prevention and treatment special line services totaled 84255 times, including 8453 graphic consultations, 59495 telephone hotline conversations, 157 remote consultations, 15510 follow-ups after consultation, and 640 business consultations (Fig. 1). The number of graphic consultations reached a maximum of 962 person-times on a single day in the early days of the outbreak. The

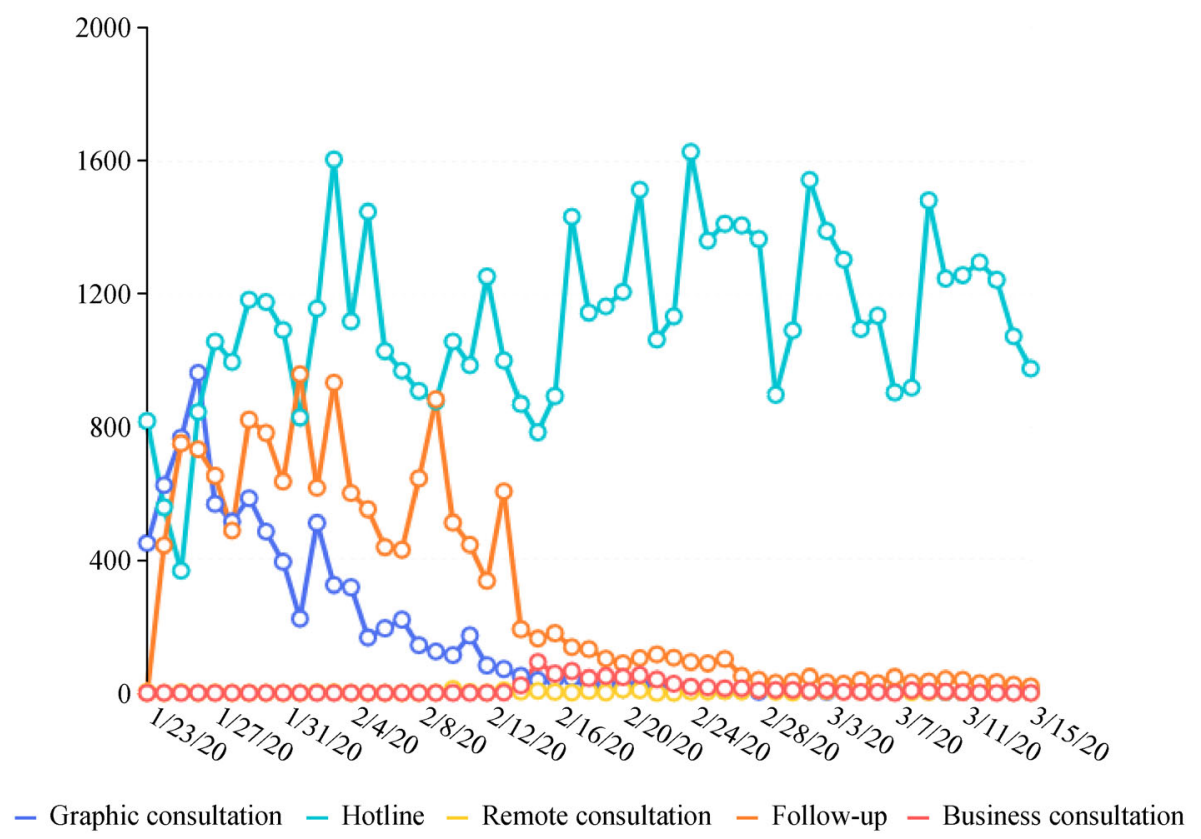

Fig. 1 Daily report of COVID-19 Special Line. 
telephone hotline has consistently accounted for the largest proportion of COVID-19 Special Line consultations, reaching a maximum value of 1603 person-times on February 3, 2020. However, the number has been fluctuating. The rest of consultation forms showed a downward trend at the end of February. From February 13th, the COVID-19 Special Line added business consultations for resumption, and it received a maximum of 93 consultations per day at the beginning. Although telemedical treatment occupies a small part of the COVID19 Special Line service, it has effectively suppressed the infection risk of hospital staff, mitigated social panic, and constructed the first anti-epidemic defense line by investigating suspected patients and conducting psychological intervention.

On the basis of follow-up service after the COVID-19 Special Line consultation, we collected follow-up data and analyzed the source and distribution of patients. We observed 15510 person-times with follow-up services, including 9347 online and offline services and 4394 follow-ups.

Moreover, a total of 4958 suspected cases (31.97\%) were divided into three categories based on their contact history and symptoms (Table 1). Exactly 170 cases with contact history were symptomatic, 1244 were asymptomatic with contact history, and 3544 were symptomatic without contact history. As of March 15, through this form, we have found 6 confirmed and 1990 suspected cases, which play an important role in the diagnosis of cases and the control of the epidemic.

In terms of treatment, 146 cases diagnosed in Taizhou, including several cases confirmed via telemedicine, have been cured and discharged. We sampled 65 discharged patients during this period, including $39(60 \%)$ men and 26 $(40 \%)$ women. A total of $10.77 \%$ of patients were younger than 30 years old, $32.31 \%$ were between 31 and 40 years old, $30.77 \%$ were $41-50$ years old, and the remaining $(26.16 \%)$ patients were over 50 years old. Farmers accounted for $61.54 \%$ of the total number of patients. Thirty-five $(53.85 \%)$ patients had symptoms of fever, 7 $(10.77 \%)$ had coughs, and 25 (38.46\%) had lung infection. The analysis of patient medications showed that $98.46 \%$ of discharged patients used lopinavir/ritonavir and $\alpha$-inter- feron nebulization, and more than $50 \%$ of patients used probiotics and oxygen inhalation treatments, which also reflect the treatment plan of integrated Chinese and Western medicine that is conducive to patient cure.

\section{Further findings and limitations of Taizhou model}

Compared with simple hospital medical education and traditional media science popularization, this innovative preventive measure combined with Internet medical technology has many natural advantages. The COVID-19 Special Line integrates a variety of Internet medical methods, such as online graphic consultation, video consultation, remote consultation, telephone consultation, WeChat group communication, and WeChat mini-program self-test. This line covers hospital-to-ordinary people and hospital-to-township health center channel forms, integrates personalized science content and medical services, and delivers urgently needed professional medical diagnosis, epidemic prevention, and control knowledge to the people through information flow. A research-supported Internet hospital can serve different types of epidemic counselees, offer essential medical supports to the public during the COVID-19 outbreak, reduce social panic, promote social distancing, enhance the public's ability of self-protection, correct improper medical-seeking behaviors, reduce the chance of nosocomial cross-infection, and facilitate epidemiological screening [1]. This condition is consistent with our results, that is, patients with common diseases quickly confirm their situation and eliminate unnecessary panic. Collectively, these measures may effectively reduce people gathering in offline hospitals [2], and having the same concept as our model can reduce the risk of cross-infection of people who use public transportation to hospitals and other densely populated places. In addition, as the best-selling form of COVID-19 Special Line consultation, hotline service can provide oneto-one online consultation to fully protect the privacy of patients, and it can also provide high-quality medical resources for people in remote areas, such as mountains and villages, across time and space limitations. Unlike

Table 1 COVID-19 follow-up cases statistics

\begin{tabular}{|c|c|c|c|c|c|}
\hline \multirow{2}{*}{ Type } & \multirow{2}{*}{ Cases } & \multicolumn{2}{|c|}{ Clinic situation } & \multirow{2}{*}{ Confirmed cases } & \multirow{2}{*}{ Suspected cases } \\
\hline & & Outpatient service & In the hospital & & \\
\hline $\begin{array}{l}\text { History of exposure, } \\
\text { symptomatic }\end{array}$ & 170 & 64 & 51 & 6 & 164 \\
\hline $\begin{array}{l}\text { History of exposure, } \\
\text { asymptomatic }\end{array}$ & 1244 & 7 & 6 & 0 & 1244 \\
\hline $\begin{array}{l}\text { No history of exposure, } \\
\text { symptomatic }\end{array}$ & 3544 & 411 & 98 & 0 & 582 \\
\hline Total & 4958 & 482 & 155 & 6 & 1990 \\
\hline
\end{tabular}


other countries who passively seek close contacts of patients by dialing telephones, the opening of telemedicine also provides people with a two-way active interaction to understand COVID-19.

Many hospitals in Zhejiang Province have successively opened internet fever consultation clinics. On February 7, 2020, a patient diagnosed with COVID-19 in Taizhou was formally cured and discharged after consultation on the Internet. This case became the first online initial screening and confirmed cured case in China. A period of 14 days lasted from the first online consultation to discharge from the hospital, which encouraged many Internet healthcare practitioners. Facing the growing trend of overseas epidemics, Taizhou Enze Medical Center (Group) has also actively shared the anti-epidemic experience of Enze with foreign medical teams such as Italy, especially the establishment of COVID-19 Special Line, which has served nearly 100000 people in 30 provinces and cities nationwide through the Internet, to eliminate inner panic for ordinary people. Professor Li Ling, a professor at Peking University and a consultant for medical reform under the State Council, also promoted the "Taizhou Experience," suggesting the full use of local and foreign Internet medical services and dedicated phone and internet platforms by using information flow instead of people flow logistics. If one can solve problems on the Internet and phone, going out is unnecessary [3].

In addition to the COVID-19 Special Line, the entire telemedicine team launched online services, such as WeChat public account, online live lectures, and remote consultation. The public can self-examine their physical condition with the help of telemedicine, mitigating the pressure of hospital diagnosis and treatment. Experts made full use of the telemedicine platform to achieve online live epidemic prevention and popularization of science to suppress the risk of cross-infection caused by the dense crowd of offline clinics and improve the hospital's social responsibility. For patients in remote areas or with chronic disease re-diagnosis needs, the hospital will contact primary health institutions for remote consultation, and the Internet will contact pharmaceutical companies and logistics companies to cooperate to start distribution services. This online consultation based on the Internet hospital can effectively cut off the transmission of the virus, reduce the risk of cross infection, and follow up all suspicious, suspected, and confirmed patients.

Taizhou has also made many efforts in offline services. With the resumption of work and production enterprises, to better protect the safety of enterprise employees and prevent the spread of cross-infection and epidemics, Taizhou has set up an online enterprise recovery control consulting service by taking advantage of the Internet medical features. Volunteer teams were organized to conduct in-depth emergency response drills for COVID-
19 in individual companies. An expert group was established to help companies develop specific solutions, such as epidemic prevention and emergency plans before returning to work, and regulate temperature monitoring, factory management, canteens, temporary isolation areas, environmental cleaning and disinfection, and other details.

Taizhou's "Internet + COVID-19 prevention and control model" (Internet + COVID-19 model) also has an impact on the Chinese medical system. In this new epidemic, the advantages of Internet medical treatment have been further tapped. Given the convenience of noncontact consultation, online medical care can meet people's daily consultation needs, such as cold and fever, without leaving home and timely relieve tensions about the disease. The Chinese government also affirmed this advantage and specifically requested administrative departments and medical institutions at all levels to actively use various channels and forms to publicize related services, increase Internet medical policy support, and promote the development of Internet medical industry.

The Internet + COVID-19 model also revealed deficiencies during the operation, that is, mainly relying on the Deming cycle to check problems and difficulties. Before the launch, patients had a low awareness of the service content of COVID-19 Special Line. Therefore, through the website, WeChat public account, and news media, COVID-19 Special Line expanded their influence and informed the public about their preference for online consultation. In the initial stage of the hotline channel after opening, the average waiting time for patient consultations exceeded $30 \mathrm{~s}$, and consultations in multiple channels were optimized by the information center. When the number of waiting patients in each channel exceeded 3, they will be automatically shunted to improve the timeliness of admission. Aside from strengthening the construction of the Internet platform, the hospital launched trainings for technicians and medical staff. In addition, based on the hospital management information system, hospital inspection information system, and hospital imaging information system, various medical institutions have established an interconnection and sharing mechanism to achieve data sharing.

\section{Conclusions}

Faced with this complicated epidemic situation, the Taizhou Internet + COVID-19 model is constantly improved, including in all details and services, to identify the source of infection and refine the control. This model includes not only the cooperation of primary health institutions at the national level but is also a breakthrough to improve medical services during this epidemic; it has 
realized the comprehensive utilization of medical resources in a fast, convenient, and efficient way. The COVID-19 Special Line service has also established a hospital, government, and society epidemic prevention system, benefiting remote islands and mountains in the country and in the region. As a result, more people feel and agree with the Internet as a new medical service model. Thus, we can believe that the Internet + COVID-19 model will provide a reference model for epidemic prevention and control for similar public health emergencies in the future.

COVID-19 has become a global pandemic, and the rapid spread of COVID-19 around the world has made online surveys an important tool for tracking public knowledge and misunderstandings during the rapid outbreak of infectious diseases in the United States and the UK [10]. Our country responded quickly, implemented appropriate measures, and made huge contributions and sacrifices. The pandemic also reflects the shortcomings in the construction of our public health system. At the same time, this phenomenon may become a catalyst for the development of telemedicine and cultivate better medical habits and safety awareness for patients. Telemedicine has various advantages, such as full information coverage, medical resource sharing, personalized customization, and efficient disease management, which will be the direction of future medical development. However, several economic, medical professional, legal supervision, and other issues that have plagued its long-term development must be solved to enable the orderly and quality development of the Internet medical industry.

\section{Compliance with ethics guidelines}

Cenyi Shao, Shijian Li, Feng Zhu, Dahai Zhao, Hui Shao, Haixiao Chen, and Zhiruo Zhang declare no conflicts of interest. This manuscript does not contain any studies with human or animal subjects and does not involve a research protocol requiring approval by the relevant institutional review board or ethics committee.

\section{References}

1. Gong $\mathrm{K}, \mathrm{Xu} \mathrm{Z}$, Cai Z, Chen Y, Wang Z. Internet hospitals help prevent and control the epidemic of COVID-19 in China: multicenter user profiling study. J Med Internet Res 2020; 22(4): e18908

2. Little P, Stuart B, Andreou P, McDermott L, Joseph J, Mullee M, Moore M, Broomfield S, Thomas T, Yardley L. Primary care randomised controlled trial of a tailored interactive website for the self-management of respiratory infections (Internet Doctor). BMJ Open 2016; 6(4): e009769

3. Ling L. Seven specific suggestions to the Wuhan Municipal Party Committee and Municipal Government. January 24, 2020. https:// user.guancha.cn $/ \mathrm{main} /$ content $? \mathrm{id}=231202 \& \mathrm{~s}=$ zwyess $($ in Chinese) (accessed March 8, 2020)

4. Geldsetzer P. Use of rapid online surveys to assess people's perceptions during infectious disease outbreaks: a cross-sectional survey on COVID-19. J Med Internet Res 2020; 22(4): e18790 